\title{
A New Search Algorithm for Reducing the Incidence of Missing Cottonoids in the Operating Theater
}

\author{
Abhinandan Reddy, Rajat Mahajan, Tarush Rustagi, Shakti A. Goel, \\ Murari L. Bansal, Harvinder Singh Chhabra \\ Department of Spine Surgery, Indian Spinal Injuries Center, New Delhi, India
}

\begin{abstract}
Study Design: Retrospective study.
Purpose: Missing cottonoids during and after spinal surgery is a persistent problem and account for the most commonly retained surgical instruments (RSIs) noticed during a final cottonoid count. The aim of this study was to enumerate risk factors and describe the sequence to look out for misplaced cottonoids during spinal surgery and provide an algorithm for resolving the problem.

Overview of Literature: There are only a few case reports on RSIs among various surgical branches. The data is inconclusive and there is little evidence in the literature that relates to spinal surgery.

Methods: This retrospective study was conducted at Indian Spinal Injuries Centre. The data was collected from hospital records ranging from January 2013 to December 2017. The surgical cases in which cottonoid counts were inconsistent during or after the procedure were included in the study. The case files along with operating theater records were thoroughly screened for selecting those in which there was confirmed evidence of such an event.

Results: There were 7,059 spinal surgeries performed during the study period. Fifteen cases of miscounts were recorded with an incidence of one in every 471 cases. Cottonoids were most commonly lost under the shoes of the surgeon or assistants. In two instances, cottonoids were found in the surgical field and trapped in the interbody cage site. Based on these locations, a systematic search algorithm was created

Conclusions: This study enumerates RSI risk factors in spinal surgical procedures and describes steps that can be followed to account for any missing cottonoids. The incidence of missing cottonoids can be decreased using a goal-oriented approach and ensuring that surgical teams work in collaboration.
\end{abstract}

Keywords: Cottonoids; Spine; Algorithms; Re-exploration; Retained foreign body; Surgical sponges

\section{Introduction}

Neurosurgical patties, commonly called cottonoids, are one of the most frequently used objects during spinal surgery [1]. Retained/missing cottonoids, although rare, is a persistent problem in these surgeries. The incidence of retained cottonoids has substantially decreased because of the efforts of the operating room staff. Discrepancies in

Received Jun 12, 2018; Revised Jul 9, 2018; Accepted Jul 16, 2018

Corresponding author: Tarush Rustagi

Indian Spinal Injuries Centre, Vasant Kunj, New Delhi-110070, India

Tel: +91-011-42255225, Fax: +91-422552000, E-mail: tarush.rustagi@gmail.com 
the final cottonoid count result in an increase in operating theater time that is spent randomly looking for the missing object and causes anxiety among surgeons and staff. Missing cottonoids can pose a potential threat to the patients in the form of neurological manifestations, re-exploration surgeries, infections, or mortality [2] and can result in subsequent financial, medico-legal consequences for the surgeon, surgical trainees, hospitals, and health care providers [3-5]. Although there are numerous studies on retained surgical instruments (RSIs), there is a paucity of literature on lost cottonoids in spinal surgery. We present our study that enumerates the risk factors and describes a systematic search algorithm to find missing cottonoids during and after major spinal surgery.

\section{Materials and Methods}

The study was approved by the Institutional Review Board of Indian Spinal Inquires Centre (IRB approval no., ISIC/ $\mathrm{RP} / 2018 / 094)$. IRB waived the requirement for informed consent. Five-year data from January 2013 to December 2017 were collected and screened. The surgical cases in which the RSI counts were incorrect during or after the procedure and an incident report was filed were included in the study. Minimally invasive surgeries, such as percutaneous procedures, were excluded from the study (Fig. 1). The case files together with operating theater records

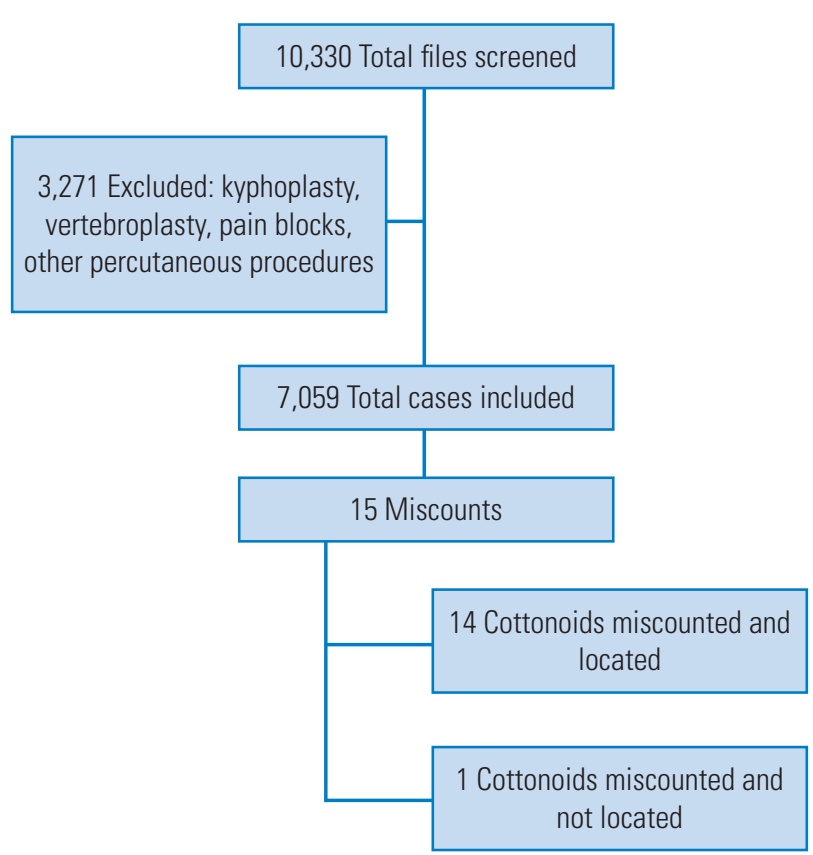

Fig. 1. Flowchart of data search and case screening. were thoroughly screened to select those in which there was confirmed evidence of such an event. The respective surgeons involved in the spinal surgeries were consulted regarding the possible risk factors and locations of the cottonoids in their respective cases.

All operating rooms had a scrub nurse/floor nurse, anesthetist, anesthesia technician, fluoroscopy machine technician, surgeon, and one or two assistant surgeons. A safe surgical checklist proposed by the World Health Organization (WHO) was followed for all surgeries performed as per hospital protocols [6]. The operating surgeon/scrub nurse was informed of the cottonoid count before commencement of the surgery and the numbers were added, if any, during the surgery. The scrub nurse was regularly updated of the number of cottonoids placed and removed within the surgical field. Two cottonoid sizes were used $(1 \mathrm{~cm} \times 2 \mathrm{~cm}$ and $2 \mathrm{~cm} \times 5 \mathrm{~cm})$.

\section{Results}

There were 7,059 spinal surgeries during the study period, which comprised posterior decompression $(n=1,301)$, discectomy $(n=1,024)$, lumbar instrumented fusion (lumbar interbody fusion, posterolateral fusion, corpectomy; $\mathrm{n}=2,098$ ), posterior cervical decompression and fusion $(n=826)$, and deformity correction $(n=149)$. Based on the definition suggested by Greenberg et al. [7], we found 15 cottonoid miscounts (all small size) in 7,059 surgeries, with a rate of one loss in every 471 surgeries (Table 1 ). Fourteen miscounts (93\%) were recorded as misplaced but were eventually traced and found in various locations in the operating room (Table 2). One cottonoid was declared lost and the wound closed after a thorough search (7\%), for a lost rate of one in 7,059 surgeries. The majority of the misplaced cottonoids were lost in surgeries associated with spinal instrumentation $(11 / 15,73 \%)$ (Table 3). Routine intraoperative fluoroscopy using an image intensifier was used to identify any missing cottonoids

Table 1. Incidence of miscounts of cottonoids

\begin{tabular}{lc} 
Variable & No. of cases \\
\hline Total cases & 7,059 \\
Miscounted cottonoids & 15 \\
Misplaced and located & 14 \\
Misplaced and not located & 1 \\
\hline
\end{tabular}


Table 2. Location where miscounted cottonoids were located ( $n=14)$

\begin{tabular}{lc} 
Places where located & $\begin{array}{c}\text { Misplaced } \\
\text { cottonoids }\end{array}$ \\
\hline Under primary surgeon shoe & 3 \\
\hline Trapped around the interbody cage & 2 \\
Under assistants shoe & 2 \\
\hline Under the wheel of the fluoroscopy machine & 2 \\
\hline Trapped in the suction machine & 2 \\
\hline Trapped in the surgeon's glove & 1 \\
\hline Found between draping towels & 1 \\
\hline Entangled in high speed burr & 1 \\
\hline
\end{tabular}

Table 3. Miscounted cottonoids and type of surgery $(n=15)$

\begin{tabular}{lc} 
Type of surgery & $\begin{array}{c}\text { Miscounted } \\
\text { cottonoids }\end{array}$ \\
\hline Lumbar interbody fusion & 5 \\
\hline Adult deformity correction & 3 \\
\hline Lumbar decompression & 3 (including one lost) \\
\hline Posterior approach based corpectomy & 2 \\
\hline Posterior cervical decompression and fusion & 1 \\
\hline Dorsal decompression & 1 \\
\hline
\end{tabular}

within the surgical field. In two cases, cottonoids were found within the surgical field, and in the remaining 12 cases, they were found outside. In the case in which the cottonoid was misplaced and never found, the surgeon closed the lumbar fusion wound after documenting the loss. On the first day after surgery, the patient complained of severe radicular pain and magnetic resonance imaging (MRI) showed a signal intensity change along the Dural sac, which raised suspicion of the presence of a foreign body. After an informed consent, the wound was re-explored, but the result was negative; however, the patient's symptoms of radicular pain were relieved. Based on these cases, we have proposed a systematic search algorithm, as depicted in Fig. 2.

\section{Discussion}

Taking counts of surgical equipment before and after surgery is an important component of the safety list protocols. This procedure involves physically counting all disposable items before the surgeon begins to close the wound. The aim is to decrease the potential of harm to the patient

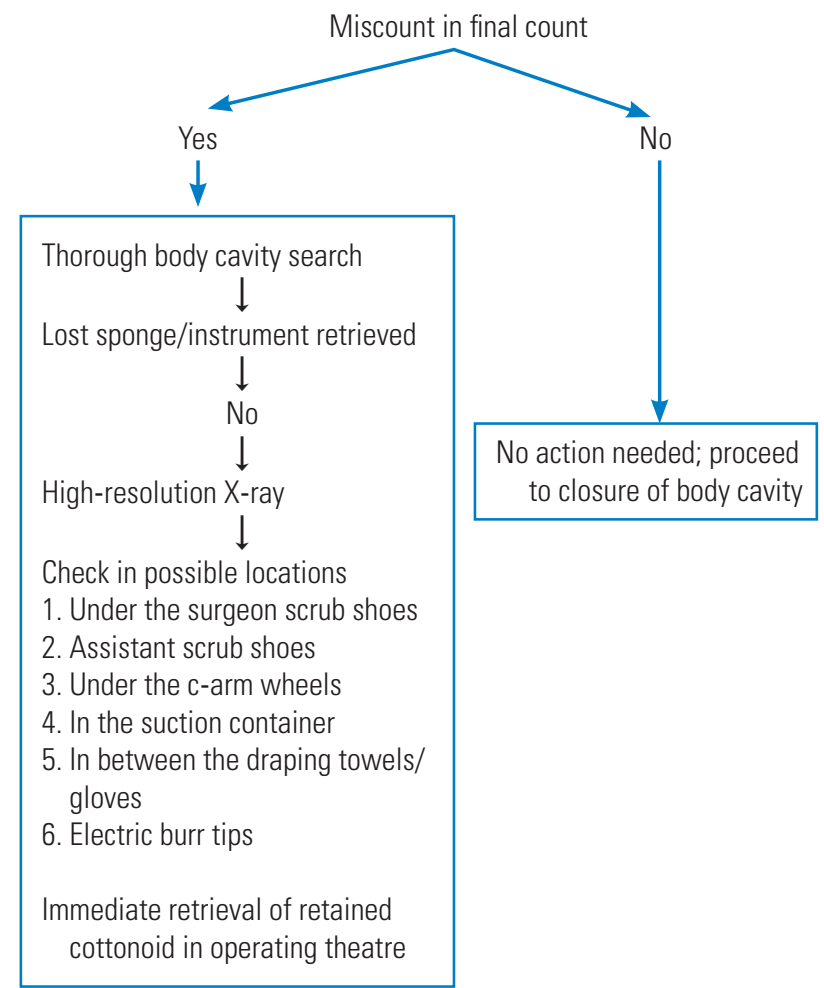

Fig. 2. Suggested algorithm to prevent retained cottonoids.

caused by incidental retention of surgical items during the procedure [8]. Retained cottonoids cause undue physical, emotional, and financial/legal consequences to both the patient and treating doctor. Many third-party bearers do not reimburse for revision surgeries for such events [9].

Greenberg et al. [7] described the following specific definitions in reporting missing surgical equipment. 'Discrepancy' is defined as "any instance in which a subsequent count does not agree with a previous one." A 'miscount' is defined as a "type of discrepancy occurring when the number of sponges, instruments, or cottonoids counted does not reflect the number of items that are actually present." A 'misplaced item' is defined as "one that is unintentionally lost in such locations as on the floor, in the trash, or in the drapes, and may or may not be subsequently relocated." A 'retained item' is defined as "a misplaced instrument that is located within the patient's body cavity before the patient leaves the operating room." In our study, there was a $0.14 \%$ incidence of cottonoid miscounts, of which most (93\%) were eventually resolved before wound closure.

We also suggest here an search algorithm that may help the staff to systematically search for the missing object rather than resorting to a time-consuming, random 
search. Most of the cottonoids in our cases were lost in surgeries that used spinal instrumentation (11/15). This loss could be associated with the extent of exposure, blood loss, and the time required for instrumented fusion compared to that required for decompression surgeries.

Schachner [10] and Crossen [11] proposed having a separate staff for counting sponges, which is a prophylactic approach for decreasing the incidence of miscounts; however, that method was not fool proof and required multidisciplinary teamwork to secure patient safety [12]. WHO in 2009 advocates a safe surgery checklist, a 10-point objective for preventing such inadvertent occurrences that are risks to the patient [6].

The incidence of RSIs is estimated to be between 1 in 5,500 and 1 in 18,760 surgeries but is unknown in spinal surgery [13]. Malpractice claims have served as a proxy data source, which most likely underreport the true incidence because of unreported asymptomatic patients, unreported near misses, and unpursued malpractice claims.

Retrospective and observational studies have estimated the incidence of RSIs as well as the risk factors [14-16]. Majority of RSI cases in published reports often fail to investigate and resolve discrepancies [7]. The existing literature lacks data on how frequently the count successfully detects any discrepancies before the patient is moved out of the operating room [15-18]. These events are classified by the Joint Commission and National Quality Forum as 'never events' $[3,4]$.

We experienced 15 such never events in the 5-year study period. In two instances, the surgical cottonoid was found within the surgical field and involved use of an interbody cage. The cottonoid had been placed over the exiting nerve root to protect it during disk preparation and it was accidentally pushed inside the disk space when the cage was inserted. We strongly recommend that the surgeon to be mindful of all cottonoids placed within the vicinity of the cage insertion and, ideally, remove them before finally inserting the cage. The primary method currently used to detect missing cottonoids within a surgical field is to take an intraoperative radiograph. In neither of the above cases could the intraoperative imaging identify the cottonoid that was stuck between the polyetheretherketone cage and endplate because the cage marker clouded the image.

One cottonoid was never located and was considered lost in spite of re-exploring the wound after surgery. This patient presented with acute radicular pain and the MRI suggested the possibility of a foreign body. Although MRI is a useful tool, it is not considered to be the gold standard for detecting a retained foreign body. An MRI has poorly sensitivity in an acute setting and is material dependent $[19,20]$. Retained sponge materials appear hypointense on T1-weighted MR images and hyperintense on T2-weighted MR images [21].

Outside the surgical field, the cottonoid was most commonly found (five of 14 cases) under the shoe of surgery staff. We suggest that this be the first place to look for any miscounted cottonoids. In a few cases, the missing cottonoids were found under the wheel of the fluoroscope, which should also be moved while looking for any missing cottonoids. Small cottonoids can be sucked through suction tips used while irrigating the surgical field or during sudden excessive bleeding. We found two such cases in which the missing count was resolved after the suction containers were searched.

This study highlights that taking a step-by-step approach to recounting and searching for missing cottonoids in suspected areas improves the incidence of resolving count discrepancies (Fig. 2). It is the responsibility of the primary surgeon to regularly update the scrub nurse regarding number of cottonoids being placed and removed throughout the surgery. The suggested algorithm can help surgeons/staff to develop a systematic approach to searching and resolving count discrepancies to avoid confusion among staff. This study may also form background for future studies.

A standard procedure for counting surgical equipment before and after surgery reduces risks and ensures continuity and efficiency. The procedure should include judicious timings of the counts, including initial and closing counts and counts when new items are added. All efforts should however be taken to avoid interruptions during crucial stages of the surgery to avoid any distraction [9].

Documentation of the any discrepancies should be thorough and appropriately investigated. Some of the recommendations are enlisted in Table 4.

The primary surgery must ensure that a wound is methodically explored and an intraoperative fluoroscopy is taken to confirm that no surgical instrument is left behind. The patient and the patient's family members should be informed about any such event and the subsequent plan for their removal. 
Table 4. Recommendations to prevent the retention of cottonoids

Recommendations
1. Using cottonoid with radio-opaque marker.
2. Communicating when placing items in the wound
3. Acknowledging the start of the count process
4. Notifying the perioperative team when items have been returned
to the field after counts are completed.
5. Count out loud and adhere to standardized counting procedures.
6. Count in the same sequence every time; and dispense dressing
sponges only after the final count has been completed.
7. Maintain an optimal operation room environment.
8. Perform a methodical wound exploration before closure of the
surgical site.

\section{Conclusions}

Lost cottonoids during spinal surgery present a serious concern and frequently account for most RSIs. After reviewing cases from 5 years of surgery records for miscounted cottonoids, we proposed a systematic search algorithm for locating misplaced cottonoids that we believe will reduce the incidence of discrepancies in cottonoid counts before and after surgery.

\section{Conflict of Interest}

No potential conflict of interest relevant to this article was reported.

\section{References}

1. Kinoshita M, Taniguchi M, Takagaki M, Seike N, Hashimoto N, Yoshimine T. Development of a nontissue adherent neurosurgical patty and an ex vivo testing system to evaluate adherent characteristics. J Neurosurg 2015;122:1180-4.

2. The Joint Commission. Sentinel event data: event type by year, 1995-second quarter 2011 [Internet]. Oakbrook Terrace (IL): The Joint Commission; 2011 [cited 2018 May 20]. Available from: https://www. jointcommission.org/se_data_event_type_by_year_/.

3. Stawicki SP, Moffatt-Bruce SD, Ahmed HM, et al. Retained surgical items: a problem yet to be solved. J Am Coll Surg 2013;216:15-22.

4. Moffatt-Bruce SD, Ellison EC, Anderson HL 3rd, et al. Intravascular retained surgical items: a multicenter study of risk factors. J Surg Res 2012;178:519-23.

5. Stawicki SP, Evans DC, Cipolla J, et al. Retained sur- gical foreign bodies: a comprehensive review of risks and preventive strategies. Scand J Surg 2009;98:8-17.

6. Freitas PS, Silveira RC, Clark AM, Galvao CM. Surgical count process for prevention of retained surgical items: an integrative review. J Clin Nurs 2016;25:1835-47.

7. Greenberg CC, Regenbogen SE, Lipsitz SR, DiazFlores R, Gawande AA. The frequency and significance of discrepancies in the surgical count. Ann Surg 2008;248:337-41.

8. Rowlands A. Risk factors associated with incorrect surgical counts. AORN J 2012;96:272-84.

9. Goldberg JL, Feldman DL. Implementing AORN recommended practices for prevention of retained surgical items. AORN J 2012;95:205-16.

10. Schachner A. VI. Foreign bodies accidentally left in the abdominal cavity: with report of one hundred and fifty-five cases. Ann Surg 1901;34:678-702.

11. Crossen HS. Operative gynecology. 2nd ed. St. Louis (MO): Mosby; 1920.

12. Asiyanbola B, Etienne-Cummings R, Lewi JS. Prevention and diagnosis of retained foreign bodies through the years: past, present, and future technologies. Technol Health Care 2012;20:379-86.

13. Hariharan D, Lobo DN. Retained surgical sponges, needles and instruments. Ann R Coll Surg Engl 2013;95:87-92.

14. Moffatt-Bruce SD, Cook CH, Steinberg SM, Stawicki SP. Risk factors for retained surgical items: a metaanalysis and proposed risk stratification system. J Surg Res 2014;190:429-36.

15. Cima RR, Kollengode A, Garnatz J, Storsveen A, Weisbrod C, Deschamps C. Incidence and characteristics of potential and actual retained foreign object events in surgical patients. J Am Coll Surg 2008;207:80-7.

16. Romano PS, Geppert JJ, Davies S, Miller MR, Elixhauser A, McDonald KM. A national profile of patient safety in U.S. hospitals. Health Aff (Millwood) 2003;22:154-66.

17. Gawande AA, Studdert DM, Orav EJ, Brennan TA, Zinner MJ. Risk factors for retained instruments and sponges after surgery. N Engl J Med 2003;348:229-35.

18. Lincourt AE, Harrell A, Cristiano J, Sechrist C, Kercher K, Heniford BT. Retained foreign bodies after surgery. J Surg Res 2007;138:170-4.

19. Ribalta T, McCutcheon IE, Neto AG, et al. Textiloma 
(gossypiboma) mimicking recurrent intracranial tumor. Arch Pathol Lab Med 2004;128:749-58.

20. Nishio Y, Hayashi N, Hamada H, Hirashima Y, Endo S. A case of delayed brain abscess due to a retained intracranial wooden foreign body: a case report and review of the last 20 years. Acta Neurochir (Wien) 2004;146:847-50.

21. Matsuki M, Matsuo M, Okada N. Case report: MR findings of a retained surgical sponge. Radiat Med 1998;16:65-7. 